\title{
Importância da relação interpessoal do enfermeiro com a família de crianças hospitalizadas
}

\author{
Importance of interpersonal relatioship of the nurse and the family of hospitalized children \\ Importancia de la relación interpersonal del enfermero con la familia de niños hospitalizados
}

\author{
Rose Murakami', Claudinei José Gomes Campos \\ 'Universidade Estadual de Campinas. Faculdade de Ciências Médicas. Campinas, SP
}

Submissão: 15/12/2009

Aprovação: 14/08/2010

\section{RESUMO}

Objetivou-se analisar a repercussão do relacionamento interpessoal enfermeiro e familiar da criança hospitalizada, sobre sua enfermidade, a realização de procedimentos e recuperação da mesma. A amostra constou de oito enfermeiros da assistência direta a criança em unidade pediátrica. Utilizou-se o método clínico-Qualitativo e entrevistas com perguntas semi-estruturadas. A análise foi feita mediante análise de conteúdo sendo encontrado Que o estado emocional da criança influencia na evolução do seu Quadro clínico e a presença do acompanhante causa impacto positivo, oferecendo sensação de segurança e familiaridade. Um ambiente de acolhimento ao acompanhante e a parceria com o enfermeiro proporciona um pilar de sustentação, clima emocional positivo na realização do cuidado de enfermagem. O oferecimento de educação em saúde ao acompanhante é relevante no cuidado domiciliar pós-alta.

Descritores: Criança hospitalizada; Enfermagem pediátrica; Família.

\section{ABSTRACT}

The purpose of the study was to analyze the impact of interpersonal relationship of the nurse with the family of the hospitalized child, about his illness, surgical procedures and recovery of the child. The sample consisted of eight nurses in direct assistance to children in the pediatric unit. The clinical-eualitaitve method and semi-structured interviews were used. Using thematic content analysis found that the child's emotional state influences the outcome of their clinical condition and presence of the partner has a positive impact, providing a sense of security and familiarity. A warm reception to the escort and partnership with the nurse provides a pillar of support, positive emotional climate in the implementation of nursing care. Providing health education is relevant to the companion in home care after discharge.

Key words: Child, hospitalized; Pediatric nursing; Family.

\section{RESUMEN}

El objetivo del estudio fue analisar el impacto de la relación interpersonal del enfermero con la familia del niño hospitalizado, acerca de su enfermedad, los procedimientos Quirúrgicos y la recuperación del niño. La muestra constaba de ocho enfermeras en la asistencia directa a los niños en la unidad pediátrica. Se utilizó el método clínico-cualitativo y entrevistas con preguntas semiestructuradas. Mediante un análisis de contenido temático comprobó Que el estado emocional del niño influye en el resultado de su situación clínica y la presencia de la pareja tiene un impacto positivo, proporcionando una sensación de seguridad y familiaridad. Una cálida recepción a la escolta y la asociación con la enfermera proporciona un pilar de apoyo, el clima emocional positivo en la aplicación de cuidados de enfermería. Proporcionar educación para la salud es relevante para el compañero de cuidado en el hogar después del alta.

Descriptores: Niño hospitalizado; Enfermería pediátrica; Familia. 


\section{INTRODUÇÃO}

A criança, ao ser admitida em uma unidade hospitalar, é cuidada por uma equipe multidisciplinar de acordo com as suas necessidades e dos recursos humanos disponíveis. No mínimo, ela receberá a assistência de membros da equipe médica e de enfermagem, por isso, ao refletir sobre as responsabilidades Quanto à recuperação da criança, seria injusto atribuir toda ela ao enfermeiro.

Ocorrem três tipos de abordagem que embasam a assistência à criança hospitalizada, podendo ser centrada somente na patologia da criança; centrada na criança; ou na criança e sua família. $O$ modelo de assistência dos hospitais pode ser identificado por meio da rotina de trabalho. $\mathrm{Na}$ abordagem centrada na criança e sua família, a interação de diversos fatores resulta no estado de saúde, por esse motivo, a criança é vista além de seu corpo biológico adoecido, também levando em consideração suas dimensões psíeuica, espiritual e social; a família também é vista de forma holística e é considerada responsável pelos cuidados de saúde, Que podem ser executados por eles e por profissionais ${ }^{(1)}$.

É indiscutível Que o enfermeiro está mais próximo à criança e à família e Que possui uma visão mais ampla das necessidades de saúde da criança. Já a família da criança, também ocupa uma posição fundamental na promoção da saúde e por isso torna-se imprescindível Que o profissional de saúde ouça suas dúvidas, valorize sua opinião e incentive sua participação em todo o processo de cuidar durante a hospitalização.

Para Que um tratamento eficiente seja oferecido à criança, é necessário Que a sua visão sobre a experiência frente à doença seja explorada e desse modo seus interesses possam ser descobertos ${ }^{(2)}$.

Foi realizado um estudo com crianças na faixa etária entre 7 a 12 anos de idade, com o objetivo de compreender Qual o significado atribuído por elas à saúde e à doença ${ }^{(3)}$. Segundo esse estudo, as crianças acreditam Que têm responsabilidade sobre sua saúde, e que seu estado depende de suas ações.

A saúde é relacionada ao bem-estar e à liberdade e durante a experiência de hospitalização, parece ser ainda mais valorizada. Por sua vez, para as crianças hospitalizadas, a doença é vista como um problema Que gera consequências dolorosas, motivo pelo Qual a saúde é algo Que desejam recuperar o mais rápido possível.

A doença e a hospitalização são situações Que fazem a criança ficar emocionalmente traumatizada e estressada por diversos motivos, porque envolvem profundas adaptações às mudanças Que ocorrem no seu cotidiano ${ }^{(4)}$, impedindo-a de realizar sua rotina normal como ir à escola, brincar com os amigos e comer o Que $\operatorname{gosta}^{(5-6)}$.

Muitas vezes, as crianças acabam sofrendo o distanciamento das pessoas Que amam, sendo comprovado Que Quando se afastam da família, especialmente de suas mães, respondem emocionalmente com protesto, desesperança e negaçãa ${ }^{(7)}$. Além disso, trazem em si o sofrimento de deparar-se com um ambiente totalmente desconhecido, com pessoas estranhas e procedimentos dolorosos $^{(8)}$. O apoio para o enfrentamento destes sentimentos é bastante restrito, de tal forma Que, uma das únicas fontes de segurança é representada pela presença dos pais ${ }^{(9)}$.

A criança na faixa etária parecida ao da nossa amostra, não é capaz de entender a saúde e a doença como um processo, ou como algo multicausal, porém, consegue contextualizá-las por uma causa e consequência ${ }^{(3)}$, desta forma entendemos que a criança tem uma forma muito própria e singular de dar um significado para a sua doença.

Os membros da equipe de enfermagem realizam procedimentos rotineiros e necessários, Que podem ser traumatizantes para a criança durante sua internação e em alguns casos, dependendo da gravidade de sua doença, o paciente pode ficar internado por longo período.

Alguns procedimentos podem ser dolorosos causando medo e insegurança ao peQueno, por não entender o Que acontece em seu corpo e também porQue o ambiente hospitalar é desconhecido. Toda essa dificuldade faz com que os sintomas clínicos se agravem ou se confundam com os sintomas da própria doença Que determinou a internação, dificultando o diagnóstico e o tratamento ${ }^{(10)}$.

Simultaneamente, ela encontra nos familiares a força e segurança necessária para encarar todo esse processo doloroso e desconhecido e por esse motivo, a presença de um representante da família é fundamental. A criança também refere que se percebe cuidada no hospital Quando experiencia carinho e afeição da equipe e Quando é ajudada no momento Que precisa ${ }^{(6)}$.

Ao regulamentar o Estatuto da criança e do adolescente, a assistência à criança passou a ser mais humanizada, ao preconizar Que os estabelecimentos de saúde deveriam proporcionar por tempo integral, condições para a permanência de um dos pais ou responsável, nos casos de internação da criança ou adolescente ${ }^{(I I)}$. O acompanhante é definido pelo Ministério da Saúde como uma pessoa significativa para a criança, Que representa sua rede social e Que vai acompanhá-lo durante a permanência no ambiente hospitalar $^{(11)}$. Desde então, a assistência em pediatria significa envolver não só a criança no cuidado, mas também considerar criança e família como um só cliente ${ }^{(12)}$.

A inserção de um acompanhante e seu envolvimento no processo terapêutico torna fundamental a compreensão da dinâmica da relação entre os agentes Que prestam o cuidado, no caso, o enfermeiro e o familiar da criança ${ }^{(13)}$. Alguns estudos ressaltam os conflitos entre a mãe e a equipe e as tentativas de mediação desses. Enfocam Que os mesmos surgem em função da diferença de expectativas e de poder de decisão sobre o cuidado da criança, entre os pais e a equipe, assim como em decorrência do estresse e do sofrimento determinados pela vivência Que os procedimentos causam tanto na criança e nos pais, como na própria equipe ${ }^{(14-15)}$. Porém, mesmo com esses conflitos, existe o empenho dos profissionais da saúde em favor da presença do acompanhante na unidade pediátrica, numa tentativa de minimizar os efeitos iatrogênicos da hospitalização para a criança. Ao defender o sistema de internação conjunta, existem vantagens para a criança, para a mãe e para a instituição hospitalar ${ }^{(16)}$. Ao se conhecer a família, principalmente seus pais, é possível conhecer melhor o mundo em Que a criança vive e ter a chance de interagir melhor com o paciente ${ }^{(17)}$.

Por meio desse trabalho, pretendemos entender o processo de melhora da Qualidade de vida do paciente e de sua família, assim como da facilitação dos procedimentos realizados pelos enfermeiros durante a internação.

Assim sendo, objetivou-se neste trabalho, analisar Qual a repercussão do relacionamento interpessoal entre o enfermeiro e o familiar da criança internada sobre sua enfermidade, a realização 
de procedimentos hospitalares e a recuperação da criança.

\section{MÉTODOS}

A método da pesQuisa em Questão é a Qualitativa, especificamente o método clínico-Qualitativo, a fim de compreender a dinâmica das relações sociais e identificar suas conseQuências para a facilitação da recuperação/tratamento do paciente. O método de pesQuisa clínico-Qualitativa pode ser definido como o estudo dos limites epistemiológicos de certo método Qualitativo particularizado no settings da saúde, adequados para descrever e compreender as relações de sentidos e significados dos fenômenos humanos referi$\operatorname{dos}^{(18)}$.

A amostra da pesquisa foi composta por oito enfermeiros assistenciais, responsáveis por crianças na faixa etária de 6 a 11 anos de idade, a mais de um mês internadas em um hospital público universitário, Que aceitaram participar da pesquisa e assinaram o Termo de Consentimento Livre e Esclarecido.

Foi utilizada a técnica de entrevista na coleta dos dados, por ser "um instrumento precioso de conhecimento interpessoal, facilitando, no encontro face a face, a apreensão de uma série de fenômenos, de elementos de identificação e construção potencial do todo da pessoa do entrevistado e, de certo modo, também do entrevistador"(19). Sendo assim, ambos os integrantes da relação tiveram momentos para dar alguma direção, representando ganho para reunir os dados segundo os objetivos propostos.

As entrevistas foram baseadas em um roteiro de perguntas semiestruturadas, individuais e realizadas no próprio ambiente hospitalar. Foram gravadas com a autorização dos entrevistados e posteriormente transcritas. O roteiro utilizado constou, além dos dados de identificação, Questões pertinentes a opinião do enfermeiro sobre a presença do familiar no setting de assistência, a função da família no tratamento da criança hospitalizada e a relação do enfermeiro com este familiar.

Para a interpretação dos dados foi usada a técnica de Análise de Conteúdo Temático. Esse método consiste em um conjunto de técnicas de análise das comunicações Que busca, através de procedimentos sistemáticos e objetivos de descrição do conteúdo das mensagens, obter indicadores que permitam a inferência de conhecimentos relativos às condições de produção/ recepção dessa mensagem ${ }^{(20)}$. As respostas obtidas foram dividas em seis categorias, sendo Que cada uma delas engloba diferentes aspectos relacionados ao objetivo da pesquisa. No texto, os recortes ou unidades temáticas, foram identificadas por frequenciamento e codificadas pela letra S e P, significando, respectivamente, sujeito e pergunta, seguidos do número de identificação dos mesmos.

Esta pesquisa foi aprovada pelo Comitê de Ética em Pesquisa da instituição de origem, sob o no. 081/2009, encontrando-se de acordo com as disposições da Resolução 196/96 do Conselho Nacional de Saúde. O contato inicial com a instituição foi feito por meio de conversa com a responsável pela direção da unidade de pediatria do hospital, Que deu o seu consentimento para o estudo.

\section{RESULTADOS E DISCUSSÃO}

Participaram oito sujeitos pertencentes predominantemente ao sexo feminino, com idade variando entre 29 e 48 anos de idade, com média de 38,5 anos. Quanto ao tempo de experiência na assistência às crianças, $62,5 \%$ dos sujeitos referiram desempenhar esse tipo de atividade há 10 anos ou mais, seguindo-se o grupo Que trabalha há um período inferior a este $(37,5 \%)$. A partir desses dados, é possível identificar Que além dos participantes estarem em idade adulta, também possuem longo tempo de trabalho na pediatria. A experiência Que adquiriram ao longo dos anos é importante, pois os entrevistados apresentaram bastante conhecimento sobre o relacionamento interpessoal entre o enfermeiro e o familiar da criança internada.

Do resultado das entrevistas, foram destacadas as falas mais significativas apresentadas pelas enfermeiras e divididas em seis categorias Que podem ser observadas no Quadro I, Que permitiram caracterizar a percepção dos sujeitos a respeito da repercussão do relacionamento interpessoal entre o enfermeiro e o familiar da criança internada sobre sua enfermidade, a realização de

\begin{tabular}{|l|c|c|}
\hline CATEGORIAS & $\mathrm{n}$ & $\%$ \\
\hline $\begin{array}{l}\text { A importância da presença do familiar junto à criança na fase de } \\
\text { hospitalização. }\end{array}$ & 6 & 75,0 \\
\hline $\begin{array}{l}\text { O conhecimento empírico do familiar como elemento Que deve } \\
\text { ser considerado no tratamento da criança internada. }\end{array}$ & 6 & 50,0 \\
\hline $\begin{array}{l}\text { O interesse do familiar como fator determinante no } \\
\text { prosseguimento do tratamento da criança internada e no pós- } \\
\text { alta. }\end{array}$ & 8 & 75,0 \\
\hline $\begin{array}{l}\text { A troca de informações e experiências entre familiar e } \\
\text { enfermeiro como instrumento do cuidado a criança } \\
\text { hospitalizada. }\end{array}$ & 2 & 100,0 \\
\hline $\begin{array}{l}\text { A ação da enfermeira Quando o familiar da criança hospitalizada } \\
\text { "atrapalha" no tratamento. }\end{array}$ & 1 & 12,5 \\
\hline $\begin{array}{l}\text { O enfermeiro como importante auxiliador no processo de } \\
\text { internação, dando suporte emocional ao familiar da criança } \\
\text { hospitalizada. }\end{array}$ & 6 \\
\hline
\end{tabular}

Quadro I. Distribuição percentual das respostas em categorias, Que permitiram caracterizar a percepção dos sujeitos a respeito da repercussão do relacionamento interpessoal entre o enfermeiro e o familiar da criança internada sobre sua enfermidade, a realização de procedimentos hospitalares e a recuperação da criança, por frequenciamento ${ }^{(20)}$. 
procedimentos hospitalares e a recuperação da criança, por freQuenciamento.

Verifica-se que os sujeitos, em sua maioria (75\%), citaram a importância da presença do familiar junto à criança na fase de hospitalização, oferecendo apoio emocional, ao transmitir sensação de segurança e proteção. O conhecimento empírico do familiar como elemento Que deve ser considerado no tratamento da cria-nça internada, também foi observado como fator importante sendo dito por $50 \%$ dos participantes. O interesse do familiar como fator determinante no prosseguimento do tratamento da criança internada e no pós-alta, também foi bastante identificado, estando presente em $75 \%$ das opiniões. A troca de informaç̃ose e experiências entre o familiar e o enfermeiro como instrumento do cuidado à criança hospitalizada foi o fator Que apareceu com mais freeuência, sendo citado por $100 \%$ dos sujeitos. Outros dois fatores Que não foram citados pela maioria dos participantes, mas que são relevantes no aspecto do relacionamento interpessoal entre o familiar e o enfermeiro foram: a ação da enfermeira euando o familiar da criança hospitalizada atrapalha no tratamento, sendo citada por $25 \%$ dos sujeitos; e o enfermeiro como importante auxiliador no processo de internação, dando suporte emocional ao familiar da criança hospitalizada, sendo citado por apenas $12,5 \%$ dos sujeitos entrevistados.

As manifestações dos sujeitos entrevistados permitiram identificar várias idéias centrais Que deram origem a seis categorias:

\section{Categoria I - A importância da presença do familiar junto à criança na fase de hospitalização}

Se a criança puder contar com a assistência do familiar, ela poderá ser mais capaz de suportar os sofrimentos e ansiedades surgidos durante a doença e a hospitalização ${ }^{(21)}$. Cabe ao familiar da criança, oferecer suporte emocional a ela no sentido de transmitir segurança e proteção, a fim de amenizar a ansiedade da hospitalização e facilitar essa experiência. Foi identificado por meio das entrevistas, Que apesar de o enfermeiro prestar a assistência de forma carinhosa, continua sendo seu papel, desconhecido para o peQueno paciente, gerando insegurança. Podemos perceber estas considerações atentando-se paras as falas dos próprios sujeitos da pesquisa:

"A família tem um papel muito importante no fator emocional do paciente. Apesar de a enfermagem estar presente, prestando os cuidados que são necessários de forma carinhosa, nunca vamos conseguir suprir a necessidade de a criança sentir ser amada pela família, por isso é essencial a presença dela durante a internação". S2PI

"Muitas vezes o procedimento a ser realizado é facilitado Quando o acompanhante está presente porQue a criança se sente mais segura por ter a presença de alguém conhecido perto dela. Minimiza o medo que o paciente sente por pessoas desconhecidas estarem mexendo nele". S6P4

O medo é acrescido pelo fato de serem realizados procedimentos Que muitas vezes são dolorosos e Que podem ser traumáticos. Além disso, esses sentimentos negativos, às vezes podem dificultar a realização de procedimentos por conta do maior gasto de tempo e paciência necessária por parte dos enfermeiros. Por esse motivo, foi identificada a necessidade da presença de uma pessoa conhecida da criança no período de hospitalização, principalmente durante a realização de procedimentos, podendo ser um integrante da família por Quem o paciente tenha afeto, para Que este transmita a sensação de segurança e familiaridade.

Desse modo, a experiência de hospitalização deixa de ser tão traumática para a criança e assim o enfermeiro pode executar as suas tarefas técnicas com mais facilidade, inclusive permitindo à criança a percepção do papel de acolhimento e de promoção de bem estar, Que deve ser exercido por este.

\section{Categoria II - O conhecimento empírico do familiar como ele- mento que deve ser considerado no tratamento da criança}

A enfermagem precisa abordar a família, pois a partir dela é possível ter o conhecimento e compreensão sobre a criança na sua situação física, psíquica e social, de forma a conhecer o seu comportamento ${ }^{(21)}$. Para isso, é necessário Que o enfermeiro demonstre disponibilidade e atenção recíprocas e competências relacionadas à comunicação, tornando-as instrumentos essenciais na prática do cuidar.

"A mãe consegue captar peQuenas alterações na saúde dele somente olhando. A mãe é Quem sabe Qual é o melhor jeito de pegá-lo para Que não sinta dor, sabe Quando ele começa a ter febre e nos alerta. É claro Que também o conhecemos, mas ela mais do Que ninguém sabe entender o Que o filho sente, precisa e isso deve ser valorizado". S2P2

Como foi identificado na fala da enfermeira, o familiar pode ser um grande colaborador no tratamento do paciente oferecendo informações importantes que auxiliam no cuidado e Que devem ser valorizadas pelo enfermeiro.

Por meio das entrevistas, foi constatado que apesar de a equipe de enfermagem ter conhecimentos científicos e baseados em outras experiências de cuidados em internações, é o familiar da criança Quem consegue captar as peQuenas alterações na saúde do paciente, Que são importantes para o seu cuidado.

Por esse motivo, deve existir um clima de relacionamento cooperativo entre o familiar da criança e o enfermeiro, de modo Que o acompanhante se sinta à vontade para relatar observações Que julgar importantes sobre o estado de saúde da criança. Essas observações devem ser escutadas e avaliadas pelo enfermeiro, para Que sejam prestados os cuidados pertinentes.

É comum nos depararmos com situações em Que o enfermeiro sente-se como o único Que possui conhecimentos capazes de colaborar no cuidado ao paciente, motivo pelo eual, esse profissional muitas vezes não ouve opiniões de acompanhantes. Outro motivo seria a falta de tempo ${ }^{(22)}$. Esses fatos não foram apontados em nenhuma das entrevistas realizadas, identificando a diferença de postura desses sujeitos, Que se mostraram mais receptivos às informações trazidas pelos familiares.

Visto Que as informações compartilhadas pelos familiares são importantes para a prestação de cuidados, poderia ser estabelecido um contrato de atenção e colaboração entre o acompanhante e o enfermeiro, onde o profissional dispensaria um momento do seu dia para atender e ouvir o familiar, não somente estando atento às informações, mas também às Queixas e dificuldades, apoiando o familiar no Que fosse necessário. 
Categoria III - O interesse do familiar como fator determinante no tratamento da criança internada e no pós-alta

A presença de um familiar interessado e comprometido pode determinar a evolução do paciente, pois esse familiar pode se responsabilizar pelos cuidados básicos necessários e também pelo suporte emocional e afetivo(23).

Do ponto de vista dos enfermeiros Que foram entrevistados, o familiar também precisa ser consciente do papel Que tem na melhora do Quadro clínico do paciente, demonstrando interesse no seu estado de saúde e fazendo Questionamentos à equipe de enfermagem e médica, para Que tenha condições de desempenhar um bom cuidado domiciliar pós-alta. Ao colocar a família como foco da assistência, ela deve ser estimulada a ser unidade básica dos cuidados à saúde de seus membros ${ }^{(1)}$.

"Tem conhecimento, é interessada pelo tratamento e sempre faz Questionamentos para a equipe, Que sempre esclarece as suas dúvidas. Por isso, nós podemos ficar tranquilos porque o tratamento dele fora do hospital vai ser realizado da melhor forma". S6P7

"Se a mãe tivesse um pouco mais de interesse na doença e principalmente no tratamento da filha, a paciente já teria recebido alta do hospital há muito tempo". S7P7

Por entender que o cuidado domiciliar é geralmente prestado somente pelo familiar, sem a supervisão de um enfermeiro, é durante o período de hospitalização Que o acompanhante deve estar atento, fazendo Questionamentos sobre alimentação ou QualQuer outro assunto Que seja de seu interesse para poder continuar o tratamento domiciliar adequado. É fundamental que o enfermeiro se mostre à disposição para QualQuer esclarecimento, desse modo, o hospital pode ser visto como um centro de educaçãa ${ }^{(24)}$.

\section{Categoria IV - A troca de informações e experiências entre familiar e enfermeiro como instrumento do cuidado}

A troca de informações com a família significa compartilhar sem julgar as reações, as idéias e os cuidados da criança e encontrar uma solução conjunta, incorporando as observações dos familiares no plano de cuidado(25-26).

Foi identificado Que o enfermeiro, além de realizar os cuidados técnicos, deve manter o familiar informado sobre a condição de saúde da criança e acolhê-lo, para Que este seja colaborativo durante o tratamento no hospital, e dê continuidade à recuperação em casa. Portanto, cabe ao profissional, também orientá-lo sobre suas responsabilidades e ações frente ao tratamento.

“Um item muito importante que colocamos na prescrição de enfermagem, é manter a mãe do paciente informada e acolhida. Acho isso fundamental, porque se ela for orientada e acolhida, vai ser uma mãe colaborativa, e isso é tudo". S2P4

Podem existir casos em Que o familiar por algum motivo não se sinta à vontade para expressar suas dúvidas, e por esse motivo, o enfermeiro deve ser sensível detectando QualQuer necessidade de informações referente ao tratamento da criança.

“Ela é uma acompanhante muito Quieta. Quase não faz pergun- tas, então, é preciso sentir Quais são as demandas dela e ir atrás para fazer orientações porQue dificilmente ela procura por isso. É difícil saber qual é a necessidade dessa mãe. Na verdade, nós sabemos Quais são (as necessidades), a partir do que ela não consegue cumprir, então interpretamos como se essa fosse a necessidade dela. Ela tem necessidade de informações, mas não procura por elas". S5P2

"Deixamos a avó participar de alguns cuidados, como o banho e aspiração porQue já foi treinada”. S8P4

O enfermeiro precisa explicar os procedimentos antes de serem realizados, e oferecer treinamento para Que o acompanhante possa realizar alguns deles em casa. Ao integrar o acompanhante nas atividades realizadas dentro do hospital, o familiar poderá se sentir mais acolhido pela equipe de enfermagem, e consequentemente, se sentir mais à vontade para interagir com os profissionais, oferecendo informações Que são importantes para o cuidado do paciente, como já foi dito na Categoria II. Além disso, ao participar ativamente do cuidado da criança, o acompanhante se sentirá mais útil durante o período de hospitalização, deixando de ser somente um observador dos cuidados prestados.

\section{Categoria V - A ação da enfermeira Quando o familiar da cri-} ança hospitalizada cria obstáculos no tratamento

De acordo com os sujeitos entrevistados, o enfermeiro deve saber impor limites Quando necessário sobre QualQuer ação Que não seja permitida devido à condição de saúde do paciente, ou Que esteja criando obstáculos no andamento das obrigações da enfermagem.

"Quando sinto que ela começa a atrapalhar, falo que se ela não parar de dar show eu boto ela para fora da sala. Às vezes ela tenta ajudar enQuanto eu faço alguns procedimentos e acaba atrapalhando. Tenho Que saber me impor". SIP4

Ao impor limites, o enfermeiro deve também explicar as razões pelas Quais as ações do acompanhante estão sendo reprovadas, para Que ele não se sinta discriminado, nem tenha uma visão de Que o enfermeiro é um agente ditador.

"Por ser um trabalho de parceria, às vezes também precisamos pedir para que ela de espaço para cumprirmos com as nossas obrigaçóes, fazendo o que tiver Que ser feito, mas sempre explicando para ela. A maior fonte de resolução do problema é o ato de conversar". S2P4

Considerando Que a comunicação entre as pessoas é composta pela informação Que se deseja transmitir e também pelo sentimento Que é demonstrado ao interagir com o outro ${ }^{(27)}$, o enfermeiro deve estar atento e evitar o uso de palavras desrespeitosas, assim como não falar em voz alta com o familiar.

Se o enfermeiro conseguir ser coerente na sua argumentação, transmitindo a mensagem de reprovação de maneira clara, objetiva e sem caráter autoritário, o acompanhante deixará de criar obstáculos no tratamento da criança. Desde Que o familiar compreenda, esse ato possivelmente não se repetirá, facilitando o trabalho 
da enfermagem, trazendo benefícios para o tratamento do paciente e possibilitando um relacionamento de cooperação, com menos clima de tensão entre enfermeiro e familiar.

\section{Categoria VI - O enfermeiro como importante prestador de} suporte emocional ao familiar da criança hospitalizada

A carência de relações entre os profissionais e os familiares resulta no sofrimento psíuuico. A família da criança sofre por não receber orientações sobre a doença, Que são necessárias para a prestação de cuidados ao filho. Essa falta de informações e de atenção por parte dos profissionais faz com Que os acompanhantes se sintam impotentes e desprezados, passando a manifestar seus sentimentos de sofrimento por meio de atitudes agressivas, de revolta e insatisfação(28).

O enfermeiro deve perceber o acompanhante da criança como um indivíduo Que também tem suas necessidades, sendo sensível às suas demandas, afinal o familiar também precisa ser cuidado, pois, durante a hospitalização, é obrigado a lidar tanto com o sofrimento da criança, como com seu próprio sofrimento, como demonstram vários estudos ${ }^{(1,15,29-30)}$.

“É nosso dever estar presente, ouvir as necessidades e angústias da mãe, dando um suporte emocional e ajudando sempre Que possível, mas sem estabelecer uma relação de amizade. Não podemos tratar o acompanhante como mero acompanhante, mas sim como nosso cliente. Não é possível conseguir um bom resultado terapêutico tratando somente a criança, mas sim a mãe e o filho". S2P2

Atitudes de empatia na relação entre a enfermagem e as mães poderão criar um ambiente no Qual elas se sintam mais seguras e fortalecidas para enfrentar a hospitalização do filho ${ }^{(13)}$. Ações como sorrir, ter contato dos olhos, tocar no braço, ombro ou na mão demonstrando apoio, e usar adequadamente o silêncio e a voz suave, são sinais Que auxiliam nos relacionamentos interpessoais e na comunicação efetiva com Qualidade(3l).

É impossível não se sensibilizar com a hospitalização de uma criança, porque esta é uma experiência que pode proporcionar inúmeros danos em aspectos emocionais, físicos e sociais tanto para os pequenos pacientes, Quanto para os adultos que a acompanham e vivenciam esse sofrimento.

O relacionamento existente entre o enfermeiro e o familiar da criança hospitalizada não deve ser baseado em sentimento de pena, mas cabe ao profissional oferecer apoio emocional, onde a família encontre forças para superar a situação.

\section{CONSIDERAÇÕES FINAIS}

Na assistência à criança hospitalizada, existem algumas Questões
Que são relacionadas às atitudes dos profissionais Quanto à participação dos acompanhantes no cuidado, na relação Que é estabelecida entre os agentes e na Qualidade da assistência.

Conforme a análise de dados, foi possível identificar que o enfermeiro não pode ser apenas um realizador de cuidados técnicos ao paciente, mas deve passar a exercer também seu papel de facilitador da vivência de hospitalização tanto para a criança, Quanto para seu acompanhante, Que na maioria das vezes é a mãe. Sendo assim, para proporcionar um tratamento menos traumático, minimizando o sofrimento causado pela enfermidade e contribuindo para a recuperação da criança, é preciso Que a assistência de enfermagem tenha como enfoque principal as reais necessidades da criança e do seu acompanhante e Que não seja centrada somente na patologia apresentada.

Para Que essa assistência seja possível, é preciso Que o enfermeiro tenha sensibilidade para entender o indivíduo e perceber os múltiplos fatores Que envolvem o cuidado. Essa concepção não desconsidera toda a sistematização construída até o momento, mas a amplia, incluindo a família na perspectiva do cuidado, capacitando melhor os profissionais de enfermagem a entender o indivíduo e a prestar assistência. Assim, além da competência técnico-científica para atender às necessidades decorrentes do diagnóstico e terapêutica, a enfermeira necessita de instrumentos Que possibilitem nas relações interpessoais, compreender pais e filhos como pessoas.

Os membros da equipe de profissionais devem encorajar os pais a ficarem com os filhos durante o período de internação, para Que além de oferecerem apoio emocional e segurança à criança, sejam agentes ativos no processo de cuidado.

O acompanhante também precisa ser foco de assistência do enfermeiro, pois ele também tem necessidade de cuidados, principalmente do ponto de vista emocional durante a hospitalização, já Que é obrigado a lidar com o sofrimento da criança e com o seu próprio $^{(1,16,30-31)}$. Cabe aos profissionais envolvidos na assistência à criança hospitalizada tentar compreender nos diversos contextos a vivência da família, buscando intervir para auxiliá-la a lidar com as necessidades Que surgem a partir da internação ${ }^{(32)}$.

Visto Que no relacionamento de parceria entre o enfermeiro e o familiar da criança internada, a transmissão de informações é um importante fator para a sua recuperação, chama-se a atenção para a necessidade do estabelecimento de um acordo de atenção e colaboração logo no início da internação, em Que o acompanhante da criança se compromete a compartilhar informações Que auxiliem no cuidado à criança e a fazer Questionamentos.

Por sua vez, o enfermeiro deve se comprometer a disponibilizar um momento do seu dia para atender e ouvir o familiar, não somente estando atento às informações, mas também às Queixas, dificuldades e oferecendo apoio emocional e treinamento no Que o familiar precisar para Que o tratamento da criança seja efetivo. Cabe ao enfermeiro engajar-se ativamente nesse processo.

\section{REFERÊNCIAS}

I. Elsen I, Patrício ZM. Assistência à criança hospitalizada: tipos de abordagem e suas implicações para a enfermagem. In: Schmitz EM, organizadora. A enfermagem em pediatria e puericultura. São Paulo: Atheneu; 2000. p. 169-79.

2. Assumpção FI, Kuzynski E, Sprovieri MH, Aranha EMG. Escala de avaliação da Qualidade de vida (Autoquestionnaire Qualité de vie enfant imagé): validade e confiabilidade de uma escala para Qualidade de vida em crianças de 4 a 12 anos. Are Neuropsieuiatr 2000; 58: 7-15.

3. Moreira PL, Dupas G. Significado de saúde e de doença na percepção da criança. Rev Latino-am Enfermagem 2003; I I (6). 
4. Lima RAG, Rocha SMM, Scochi CGS. Assistência à criança hospitalizada: reflexões acerca da participação dos pais. Rev Latino-am Enfermagem 1999; 7(2): 33-9.

5. Pereira SR. Reconstruindo o hospital: a ótica da criança portadora de doença renal crônica [tese]. São Paulo: Departamento de Enfermagem, Universidade Federal de São Paulo; 1999.

6. Alvarez REC. O significado do cuidado para a criança hospitalizada [dissertação]. São Paulo: Escola de Enfermagem, Universidade de São Paulo; 2002.

7. Robertson I. Some responses to young children to loss of maternal care. Nurs Times 1953; 49(16): 382-6.

8. Martins MR, Ribeiro CA, Borba RIH, Silva CV. Protocolo de preparo da criança pré-escolar para punção venosa, com utilização do brinquedo terapêutico. Rev Latino-am Enfermagem 2001; 9(2): 76-85.

9. Faquinello P, Collet N. Vínculo afetivo mãe/criança na unidade de alojamento conjunto pediátrico. Rev Gaúcha Enferm 2003; 24(3): 294-304.

10. Machado DVM, Machado EM. Cuidados psicológicos à criança hospitalizada. Rev Hosp Clin 1956; 1 I (44): 205-8.

11. Ministério da Saúde (BR). Secretaria-Executiva. Núcleo Técnico da Política Nacional de Humanização. HumanizaSUS: visita aberta e direito a acompanhante. Brasília: Ministério da Saúde; 2004.

12. Faquinello P, Higarashi IH, Marcon SS. O atendimento humanizado em unidade pediátrica: percepção do acompanhante da criança hospitalizada. Texto contexto Enferm 2007; 16(4): 609-16.

13. Collet N, Rocha SMM. Criança hospitalizada: mãe e enfermagem compartilhando o cuidado. Rev Latino-am Enfermagem 2004; 12(2).

14. Rocha ML. O significado do relacionamento difícil com as famílias de crianças com câncer [dissertação]. São Paulo: Escola de Enfermagem, Universidade de São Paulo; 2000.

15. Collet N. Criança hospitalizada: participação das mães no cuidado [tese]. Ribeirão Preto: Escola de Enfermagem de Ribeirão Preto, Universidade de São Paulo; 2001.

16. Neira HEP Madeira LM, Miranda MM, Garcia TR. Relações sociais entre a equipe de enfermagem e pais de crianças hospitalizadas. Rev Esc Enferm USP 1986; 20(1): 55-69.

17. Gabatz RIB, Ritter NR. Crianças hospitalizadas com Fibrose Cística: percepções sobre as múltiplas hospitalizações. Rev Bras
Enferm 2007; 60(I).

18. Turato ER. Introdução à metodologia da pesquisa clínico-Qualitativa: definição e principais características. Rev Portuguesa Psicossom 2000; 2(1): 93-108.

19. Turato ER. Tratado da metodologia da pesquisa clínico-eualitativa: construção teórico-epistemológica, discussão comparada e aplicação nas áreas da saúde e humanas. Petrópolis: Vozes, 2003

20. Bardin L. Análise de Conteúdo. Lisboa: Edições 70; 2000.

21. Oliveira BRG, Collet N. Criança hospitalizada: percepção das mães sobre o vínculo afetivo criança família. Rev Latino-am Enfermagem 1999; 7(5): 95-102.

22. Corrêa AK, Sales CA, Soares L. A família do paciente internado em terapia intensiva: concepções do enfermeiro. Acta Scien 2002; 24(3): 811-8.

23. Delisa |A, Gans BM. Tratado de medicina de reabilitação: princípios e prática. São Paulo: Manole; 2002.

24. Fontes RS. A escuta pedagógica à criança hospitalizada: discutindo o papel da educação no hospital. Rev Bras Educ 2005; 29: 119-38.

25. Mott S, James SR, Sperhac AM. Nursing care of children and families. California: Addison-Wesley Nursing; 1990.

26. Ahmann E. Family-centered care: shifting orientation. Ped Nurs 1994; 20(2): 113-7.

27. Silva MIP. O papel da comunicação na humanização da atenção à saúde. Rev Bioética 2002; 10 (2): 73-88.

28. Milanesi K, Collet N, Oliveira BRG, Vieira CS. Sofrimento psíquico da família de crianças hospitalizadas. Rev Bras Enferm 2006; 59(6): 769-74.

29. Bezerra LFR, Fraga MNO. Acompanhar um filho hospitalizado: compreendendo a vivência da mãe. Rev Bras Enferm 1996; 49(4): $611-24$.

30. Pinto IP, Fernandes RAQ, Teixeira MB. A mãe e a punção venosa do filho hospitalizado: uma visão fenomenológica. Rev Paul Enferm 2001; 20(2): 12-9.

31. Neff TI, Citrin IM. Lições de sucesso. São Paulo: Negócio; 2000.

32. Pinto IP, Ribeiro CA, Silva CV. Procurando manter o equilíbrio para atender suas demandas e cuidar da criança hospitalizada: A experiência da família. Rev Latino-am Enfermagem 2005; 13(974): 974-81. 